\title{
ZUR DATIERUNG DER ARBEIT VON KARL MARX AM II. UND III. BUCH DES KAPITAL*
}

Die fortschreitende Publikation der neuen MEGA gibt der Marx-EngelsForschung starke Anregungen. Die Bände der Zweiten Abteilung ermöglichen es uns, das Hauptwerk von Marx eingehender zu untersuchen und tiefer zu verstehen. Zugleich wird in vielen interessanten Abhandlungen, die von den Bearbeitern veröffentlicht werden, eine Reihe wichtiger Fragen sowohl der Entstehungsgeschichte als auch des theoretischen Gehalts des Kapital geklärt, wodurch unser Wissen darüber ebenfalls erweitert wird.

Unter diesen Abhandlungen ist besonders bemerkenswert: Larissa Miskewitsch, Michail Ternowski, Alexander Tschepurenko, Witali Wygodski, ,Zur Periodisierung der Arbeit von Karl Marx am ,Kapital' in den Jahren 1863 bis 1867". Die Autoren haben eingehend die Entstehungsgeschichte des II. und III. Buches des Kapital, welche bisher gegenüber der des I. Buches relativ wenig untersucht wurde, behandelt; sie haben sie in Umrissen erhellt und dadurch eine Grundlage für weitere Forschungen in diesem Bereich geschaffen. ${ }^{2}$

* Ich danke hier dem Internationalen Institut für Sozialgeschichte in Amsterdam, das mir den Zugang zu seinem Archiv gewährt hat, namentlich Herrn G. Langkau und Frau U. Balzer, die mir bei meiner Arbeit behilflich waren; ferner dem Institut für Marxismus-Leninismus beim ZK der KPdSU, das mir ebenfalls die relevanten Materialien zugänglich gemacht hat. namentlich Frau L. Mis'kevič, Herrn V. Vygodskij und Frau I. Antonova, die mich dort unterstützt haben.

1 L. Miskewitsch, M. Ternowski, A. Tschepurenko, W. Wygodski, „Zur Periodisierung der Arbeit von Karl Marx am .Kapital' in den Jaren 1863 bis 1867", in: Marx-EngelsJahrbuch, Bd 5 (1982), S. 294-322. Der Inhalt dieser Abhandlung war schon in den folgenden zwei Abhandlungen in russischer Sprache veröffentlicht: V. Vygodskij, L. Mis'kevič, M. Ternovskij, A. Cepurenko, ,O periodizacii raboty K. Marksa nad ,Kapitalom'v 1863-1867 gg.", in: Voprosy Ekonomiki, 1981, Nr 8, S. 97-107; A. Ju. Cepurenko, ..K voprosu o datirovke I-IV rukopisej vtoroj knigi ,Kapitala' K. Marksa”, in: Naučnye soobščenija i dokumenty po marksovedeniju, Moskau 1981, S. 78-97.

2 In Japan hat Kinzaburo Sato schon im Jahr 1971 die folgende Auffassung vertreten: ..Die Disposition des .Kapitals' in vier Büchern, wovon die ersten drei den ,theoretischen 
Einzelne Punkte scheinen mir noch durchaus fraglich. Ich habe in den Jahren 1980-82 im Internationalen Institut für Sozialgeschichte die Manuskripte des II. und III. Buches des Kapital selbst untersucht, wobei ich in einigen Punkten zu abweichenden Resultaten gelangt bin. ${ }^{3}$ Zwei dieser Punkte möchte ich hier erörtern.

\section{Das „Manuskript IV” des II. Buches und das davor geschriebene Fragment}

Zunächst muß ich auf ein Problem hinsichtlich der Quellen hinweisen, auf die sich die genannten vier Autoren stützen. Nachdem dieselben die Datierung des „Manuskripts IV" des II. Buches behandelt haben, schreiben sie:

Noch ein Manuskript, das zum II. Buch gehört, blieb erhalten. Es wurde von Marx nicht numeriert und von Engels im Vorwort zum zweiten Band des „Kapitals” nicht erwähnt. Wie sich erwies, ist es der von Marx ins reine geschriebene Anfang des I. Kapitels des „Manuskripts IV”.

In seinem Text gibt es keinerlei Hinweise - auch keine indirekten -, die eine ausreichend genaue Bestimmung seiner Erarbeitung gestatten. Jedoch ist dem Text des Manuskripts ein nicht numerierter Bogen beigelegt wahrscheinlich ein Umschlag -, auf dem Marx mit Tinte vermerkt hat ,15 oder 16-t? Tussy's Geburtstag?” sowie mit Bleistift „Tschernyschewski 1864 condamné aux mines. Flerowski". ${ }^{4}$

Anscheinend verwechseln die Autoren hier zwei Manuskripte. Nach meiner Untersuchung der Materialien sollte das von den Autoren ,der von Marx ins reine geschriebene Anfang des I. Kapitels des ,Manuskripts IV”" genannte Manuskript, samt seinem Umschlag, in Wirklichkeit den Anfang des ,Manuskripts IV" selbst bilden, während die ersten vier Seiten des von ihnen „Manuskript IV” genannten Manuskripts ihrerseits ein vor dem „Manuskript IV" geschriebenes, davon zu unterscheidendes Fragment darstellen. Beide Manuskripte befinden sich im IISG.

In dem neuen, heute gebrauchten Inventar des Marx-Engels-Nachlasses

Teil' und das vierte den ,historisch-literarischen' Teil bilden, war schon in der früheren Periode von Sommer 1863 bis Ende 1864 festgestellt, und zwar schrieb Marx nach dieser Disposition in ungefähr zweieinhalb Jahren von Sommer 1863 bis Ende Dezember 1865 das Manuskript des I. Buches, das aus 6 Kapiteln besteht, das ,Manuskript I' des II. Buches, das aus 3 Kapiteln besteht, und das ,Hauptmanuskript' des III. Buches, das aus 7 Kapiteln besteht, nämlich das Manuskript des ,theoretischen Teils' des ,Kapitals' in 3 Büchern." Kinzaburo Sato, „Über das Manuskript des III. Buches des ,Kapital', 2" (japanisch), in : Shiso (Tokio), Nr 564 (1971).

3 Darauf bin ich schon in einem in japanischer Sprache publizierten Artikel eingegangen: Teinosuke Otani, „Über das ,Manuskript I' des III. Buches des ,Kapitals”, in: Keizai-Shirin (The Hosei University Economic Review). Jg. 50 (1982).

${ }^{4}$ Miskewitsch, Ternowski, Tschepurenko, Wygodski, „Zur Periodisierung”, a.a.O., S. 314. 
ist das „Manuskript IV” unter der Signatur A 65 folgendermaßen registriert:

A 65 Das Kapital II, „,Der Cirkulationsprocess des Kapitals”, Manuskript IV,

zw. 1867/70, deutsch, englisch, französisch, 34 S., fol.

Das Manuskript besteht aus 34 Folioblättern (68 Seiten) im Format $203 \times$ $321 \mathrm{~mm}$ der gleichen Papiersorte, die das Wasserzeichen paralleler Linien im Abstand von $27 \mathrm{~mm}$ aufweist. Ein einmal gefalteter Bogen (zwei Folioblätter) bildet den Umschlag, dessen vier Seiten nicht paginiert sind. 15 Blätter sind fortlaufend von 1 bis 58 paginiert, jedoch gibt es außer Seite 49 noch die Seite 49 a; die letzte, nicht paginierte Seite ist leer. Ein einmal gefalteter Bogen (zwei Folioblätter) ist zwischen die Seiten 4 und 5 eingeschoben. Nur die erste der vier Seiten dieses Bogens ist beschrieben; sie trägt ebenfalls die Seitenzahl „5”. Die übrigen drei Seiten sind leer. Bei einem Vergleich des Texts der beiden Seiten 5 ist leicht zu erkennen, daß der Text der eingefügten eine Neufassung des Texts der ursprünglichen Seite 5 ist. Daraus läßt sich schließen, daß dieser Bogen von Marx später nur eingeschoben wurde, um Seite $5 \mathrm{zu}$ überarbeiten. Von den vier Seiten des Umschlags ist nur die erste beschrieben. Sie trägt die mit Bleistift geschriebene Manuskriptnummer „IV)”. Daneben steht der Vermerk: ,15 od. 16'? Tussy's Geburtstag?" Parallel zum rechten Rand ist geschrieben: „Tschernyschewski 1864 condamné aux mines. Flerowski." Auf allen beschriebenen Seiten ist mit Bleistift der Vermerk „NT” angebracht, wahrscheinlich beim Anfertigen der Photokopien für das Marx-Engels-Institut in Moskau in den zwanziger Jahren.

Wo gibt es nun im IISG das Manuskript, das der ,ins reine geschriebene Anfang des I. Kapitels des ,Manuskripts IV"' sein soll? Um es zu entdecken, muß man sorgfältig alle im IISG aufbewahrten Manuskripte des II. Buches prüfen; denn der Inventar gibt keinen Hinweis.

Im IISG ist auch das „Manuskript V” des II. Buches aufbewahrt. Es ist im Inventar unter der Signatur A 66 folgendermaßen registriert:

A 66 Das Kapital, Bd Il, Manuskript V: „Der Kreislaufsprocess des Kapitals",

1877, deutsch, englisch, französisch, 441/4 S.

In der Tat sind hier drei ganz verschiedene Manuskripte zusammengefaßt, von denen in Wirklichkeit nur das erste das „Manuskript $V$ ” ist, auf dessen Umschlag Engels den Vermerk „Manuscr. V. (1875 oder später)” schrieb. Das zweite ist ein vor dem „Manuskript V" geschriebenes Fragment, das zwei Anfänge des 1. Kapitels des 1. Abschnitts enthält und auf das Engels „Zu Ms. V. Erste Anfänge” schrieb. 
Das dritte war im alten Inventar unter der Signatur A 47 folgendermaßen registriert:

A 47 .Zweites Buch. Erstes Kapitel. (Ms. VI.l.") $4 \mathrm{~S}$. fol.

Außer dieser Beschreibung gibt es nichts, was auf irgendeine Beziehung zu einem anderen Manuskript deutete. Das Manuskript besteht aus einem einmal gefalteten Bogen im Format $210,5 \times 343 \mathrm{~mm}$, der das Wasserzeichen paralleler Linien im Abstand von $28 \mathrm{~mm}$ aufweist. Alle vier Seiten sind beschrieben, und auf allen Seiten ist mit Bleistift der Vermerk ,NV" angebracht. Obwohl es jetzt bei dem „Manuskript V" verwahrt wird, läßt sich aus der Überschrift auf der ersten Seite und aus Marx' Schreibstil schließen, daß es weder zu "Manuskript V" noch zu „Manuskript VI" gehört. Die Überschrift lautet:

Zweites Buch. Der Cirkulationsproze $B$ des Kapitals.

Erstes Kapitel. Die Cirkulation des Kapitals.

1) Die Metamorphosen des Kapitals.

Hier ist also das II. Buch noch nicht in Abschnitte, sondern in Kapitel eingeteilt. Ferner schreibt Marx dieses Manuskript in deutscher Schrift, nicht in lateinischer wie die Manuskripte ab dem „Manuskript V”. Bei einem Vergleich mit den Manuskripten I bis IV ergibt sich, daß es vor dem „Manuskript IV” geschrieben sein muß, nämlich der Anfang des „Manuskripts IV" die Neufassung dieses Fragments ist. Das für jenes benutzte Papier ist nach Qualität und Format von dem des Fragments deutlich verschieden. Es ist kaum anzunehmen, daß im IISG einige Seiten vertauscht worden wären.

Aus dem Gesagten kann man nur schließen, daß

1) der von den Autoren als ,ein nicht numerierter Bogen [...] - wahrscheinlich ein Umschlag”' genannte Bogen eben der erwähnte Umschlag des „Manuskripts IV” selbst ist,

2) auch ,der von Marx ins reine geschriebene Anfang des I. Kapitels des ,Manuskripts IV"' - aller Wahrscheinlichkeit nach von vier Seiten - der Anfang des „Manuskripts IV” selbst ist,

3) die vier von den Autoren für den Anfang des ,Manuskripts IV” gehaltenen Seiten ein vom „Manuskript IV” zu unterscheidendes, vorher entstandenes Fragment darstellen, wobei es sich um das dritte der drei im IISG unter „A 66 Manuskript V” zusammengefaßten Manuskripte handelt.

Wahrscheinlich sind die Photokopien des Umschlags und Anfangs des „Manuskripts IV" mit denen des Fragments zu irgendeiner Zeit im IML in Moskau verwechselt worden. 
Diese Schlußfolgerung besagt nicht, daß die Datierung des „Manuskripts IV” zu revidieren wäre. Jedoch entfällt das Problem der „Datierung der unvollendet gebliebenen Bearbeitung des I. Kapitels des ,Manuskripts IV". ${ }^{5}$ Die vier Autoren stützen sich bei der Datierung des ,Manuskripts IV" auf den „Hinweis auf den ersten Band des ,Kapitals”" auf „Seite 1" sowie den „Hinweis auf ,p. 188 und sqq." auf ,Seite 2". ${ }^{6}$ Da diese Seiten aber in Wirklichkeit nicht zum „Manuskript IV”, sondern zum Fragment gehören, betrifft der Schluß der Autoren nicht jenes, sondern dieses. So ist das Problem der Datierung des Fragments gelöst. Was die Datierung des „Manuskripts IV” angeht, läßt die Verwechslung der zwei Manuskripte den Schluß der Autoren aus einem Zitat von Seite 553 des ersten Bandes des Kapital auf Seite 51 des ,Manuskripts IV" unberührt; denn diese Seite 51 gehört tatsächlich zum „Manuskript IV”.

Daher gilt der folgende Schluß der vier Autoren unverändert nicht nur für die Datierung des „Manuskripts IV”, sondern auch für die des davor geschriebenen Fragments: „Das gesamte ,Manuskript IV'kann man somit der Zeit von Juni bis August 1867 zuordnen."7 Die beiden Manuskripte wurden also von Marx in dieser Zeit geschrieben. Diese Reihenfolge und Datierung der beiden Manuskripte voraussetzend, gehen wir zum zweiten strittigen Punkt über.

\section{Der Plan für das II. Buch und das „Manuskript I” des II. Buches}

Die vier Autoren sind auf Grund ihrer vergleichenden Analyse des „Hauptmanuskripts” des III. Buches und des „Manuskripts I” des II. Buches zu dem Schluß gelangt, daß Marx, die Arbeit am III. Buch unterbrechend, das ,Manuskript I" des II. Buches fertigschrieb, wonach er zur Arbeit am III. Buch zurückkehrte. So überzeugend die Annahme der Unterbrechung begründet ist, so fraglich scheint mir ihre Annahme hinsichtlich der Stelle im Manuskript des III. Buches, wo Marx seine Arbeit unterbrochen haben soll.

Die Autoren meinen, daß Marx die Abfassung des Manuskripts des dritten Buches frühestens auf Seite 256, spätestens auf Seite 275 unterbrach. Warum frühestens auf Seite 256?

Auf den Seiten 243 und 256 des Manuskripts des III. Buches verweist Marx auf den Abschnitt 3 im I. Kapitel des „Manuskripts I”, der den Zirkulationskosten gewidmet ist. Hieraus geht hervor, daß Marx sich auf den Plan des II. Buches bezog, da die Zirkulationskosten im „Manuskript I” im 
Abschnitt 4 behandelt werden. Das bedeutet unserer Ansicht nach, daß Marx die Arbeit am III. Buch frühestens auf Seite 256 unterbrach. ${ }^{8}$

Und warum spätestens auf Seite 275?

Auf Manuskriptseite 38 dieses II. Buches schreibt Marx, daß die Behandlung der Frage, wie Gold und Silber als Geldkapital funktionieren, zum IV. Kapitel des III. Buches gehört, äußert aber Zweifel, ob diese Frage überhaupt im „Kapital” dargelegt werden wird. Indes taucht sie im Manuskript des III. Buches auf den Seiten 275 bis 278 im IV. Kapitel auf. Das heißt, daß Marx zu dem Entschluß kam, die Arbeit am Manuskript des III. Buches zu unterbrechen und das II. Buch niederzuschreiben. Das erfolgte, bevor die Seiten 275 bis 278 des Manuskripts des III. Buches entstanden sind. Mit anderen Worten, Marx verfaßte das „Manuskript I” in der Zeit, die zwischen der Abfassung der Manuskriptseiten 256 und 275 des III. Buches lag. ${ }^{9}$

Prüfen wir hier zunächst die erstere Schlußfolgerung auf ihre Richtigkeit. Diese Behauptung ist nur aufrechtzuerhalten, wenn der Plan des II. Buches tatsächlich vor der Niederschrift des „Manuskripts I” des II. Buches entstanden ist. Die Autoren schreiben:

Den Plan für das II. Buch hatte Marx offensichtlich vor Beginn der Arbeit an diesem Buch auf einem Extrablatt entworfen:

Erstes Kapitel. Die Zirkulation des Kapitals.

Zweites Kapitel. Der Umschlag des Kapitals.

Drittes Kapitel.

Die ersten beiden Kapitel sind in diesem Plan in Paragraphen unterteilt. Das dritte Kapitel erhielt bei der Herstellung des ",Manuskripts I" des II. Buches den Titel „Zirkulation und Reproduktion"; gleichzeitig erarbeitete Marx die Struktur dieses Kapitels. ${ }^{10}$

Ist es aber offensichtlich, daß der Plan vor Beginn der Arbeit am II. Buch entworfen worden ist? Nein, umgekehrt! Es ist vielmehr anzunehmen, daß der Plan nach dem Abschluß des „Manuskripts I” entworfen wurde. Betrachten wir zunächst die Überschriften der ersten beiden Kapitel im Text des „Manuskripts I”.

Zweites Buch. Der Circulationsproceß des Capitals.

Erstes Capitel. Der Umlauf des Capitals.

1) Die Metamorphosen des Capitals.

2) Die Circulationszeit.

3) Die Productionszeit.

4) Circulationskosten.

8 Ebd., S. 308.

9 Ebd.

10 Ebd., S. 300. 
Zweites Capitel. Der Umschlag des Capitals.

1) Umlaufszeit u. Umschlag.

2) Fixes u. circulirendes Capital. Umschlagscyclen. Continuität d. Reproductionsprocesses.

3) Umschlag u. Werthbildung. ${ }^{11}$

Dagegen schrieb Marx den Plan für das II. Buch ,auf einem Extrablatt” folgendermaßen:

Zweites Buch. Der Cirkulationsprozeß des Kapitals.

Erstes Kapitel. Die Cirkulation des Kapitals.

1) Die Metamorphosen des Kapitals: Geldkapital, Produktives Kapital, Waarenkapital.

2) Produktionszeit und Umlaufszeit.

3) Die Cirkulationskosten.

Zweites Kapitel. Der Umschlag des Kapitals.

1) Begriff des Umschlags.

2) Fixes Kapital und Cirkulirendes Kapital. Umschlagscyclen.

3) Einfluß d. Umschlagszeit auf Produkt- u. Werthbildung u. Produktion d. Mehrwerths.

Drittes Kapitel. ${ }^{12}$

Wenn man diese beiden Versionen sorgfältig vergleicht, kann man folgende orthographische Verschiedenheiten bemerken:

„Circulationsproceß” gegen „Cirkulationsprozeß”;

„Capital” gegen ,Kapital”;

„Capitel” gegen ,Kapitel”;

„Circulation" gegen „Cirkulation";

„Productionszeit” gegen „Produktionszeit”;

„Circulationskosten” gegen „Cirkulationskosten”;

„circulirendes Capital” gegen „Cirkulirendes Kapital”.

Sowohl im „Manuskript I" und im „Manuskript III” des II. Buches als auch im „Hauptmanuskript”13 des III. Buches benutzte Marx die erste Form, d.h. schrieb er "Circulation” statt „Cirkulation” usw. Aber im

11 Institut für Marxismus-Leninismus, Zentralparteiarchiv, Moskau, f. 1, op. 1, d. 1802 ,

1. 2, 23, 25 verso, 29, 31, 35 verso, 51. Nach den Photokopien des Manuskripts, die das IML beim ZK der KPdSU dem Otsuki-Verlag zur Verfügung stellte.

12 Ebd., 1. 1.

13 Engels schreibt im Vorwort zum dritten Band des Kapitals über das Manuskript des III. Buches: „Für das dritte [Buch] lag eben nur ein, noch dazu äußerst lückenhafter, erster Entwurf vor." (K. Marx, F. Engels, Werke, Bd 25, Berlin 1964, S. 8) Aber danach bezeichnet er es als „Hauptmanuskript” (ebd., S. 11-12), da es noch drei andere kleine Manuskripte (,Ms. II”, ,Ms. III” und „Ms. IV”) gibt, wovon jedes den Anfang des I. Kapitels „Kostpreis und Profit” bildet. Engels gibt in seinem Vorwort zwei davon (,Ms. II" und „Ms. III”) an, die er für das 1. Kapitel benutzte: „zwei Ansätze einer Umarbeitung [... . jeder von 8 Seiten Folio" (ebd., S. 12). 
„Manuskript IV”, im davor geschriebenen Fragment und im „Manuskript HI" bediente er sich der zweiten Form, d.h. schrieb er „Cirkulation” statt „Circulation" usw. Meines Erachtens fällt der Ubergang in die Zeit der Vorbereitung der Druckvorlage für die 1. Auflage des ersten Bandes des Kapital bzw. der Korrektur derselben. Denn Marx mußte in dieser Auflage einheitlich die letztere Schreibweise benutzen. ${ }^{14}$ Es ist jedenfalls nicht einfach, sich vorzustellen, daß Marx vor dem „Manuskript I" des II. Buches diese Schreibweise benutzt hätte. Nach diesem Unterschied in der Schreibweise wäre also der Plan frühestens nach dem „Manuskript III” des II. Buches entstanden.

Vergleichen wir nun den Plan und das „Manuskript I” in inhaltlicher Hinsicht.

Erstens. Wenn der Plan früher, das Manuskript später geschrieben worden wäre, müßte der Abschnitt ,2) Produktionszeit und Umlaufszeit" des 1. Kapitels des Plans nachher im Manuskript in zwei Abschnitte, nämlich ,2) Circulationszeit" und ,3) Die Productionszeit”, unterteilt worden sein. Bemerkenswert ist jedoch die Tatsache, daß Marx im Manuskript bei Abschnitt 3 zunächst die Überschrift „Der Umschlag des Capitals" geschrieben hatte, die er erst danach in "Die Productionszeit” veränderte. ${ }^{15}$ Man kann in dieser Veränderung nicht einfach die bloße Korrektur eines Schreibfehlers sehen. In der Fußnote auf Seite 49 dieses Abschnitts 3 schreibt Marx:

Es ist d. Frage, ob dieser ganze $\$ 3$ d. ersten Capitels sich nicht einfach zu beschäftigen mit d. Produktionszeit, in derselben Weise, wie sich §2) mit d. Circulationszeit beschäftigte? So daß d. ch. II), was ohnehin heißt: „Der Umschlag d. Capitals" alles auf diese bestimmte Form seines Umschlags enthielte, u. Capitel 1), welches auch d. „Umlauf d. Capitals" heißt, nur d. allgemeinen Momente desselben zu analysiren? Dieß scheint allerdings $d$. Beste. ${ }^{16}$

Also hatte Marx im Abschnitt 3 nicht nur die eigentümlichen Probleme der Produktionszeit behandelt, sondern zunächst auch die allgemeine Bestimmung des Umschlags des Kapitals gegeben. Aber in jener Fußnote

14 Allerdings tat Marx dies in der 1. Auflage des ersten Bandes noch nicht ganz konsequent. Z.B. schreibt er bis zum Abschnitt 2 des 2. Kapitels „Circulation”, aber vom Abschnitt 3 desselben ab sowie im Vorwort und Inhaltsverzeichnis „Cirkulation”, was für mich bemerkenswert ist. Ubrigens ist es auch interessant, daß in der in Fraktur gedruckten Schrift Zur Kritik der Politischen Oekonomie. Erstes Heft (1859) schon die Schreibweise „Kapital”, „Kapitel”, „Cirkulation”, „Produktion” usw. benutzt worden ist.

15 Manuskriptseite 46, IML, f. 1, op. 1, d. 1802, 1. 25 verso.

16 Ebd., 1. 27. 
wollte er, darüber reflektierend, den Begriff des „Umschlags” zu Beginn des 2. Kapitels „Der Umschlag d. Capitals” klarmachen. Am Anfang des Abschnitts ,1) Umlaufszeit u. Umschlag" des 2. Kapitels schrieb er dementsprechend: „Es ist nun das im vorigen Capitel sub. 3) Anticipirte über d. allgemeinen Begriff d. Umschlags hierher zu setzen"17; was die Umstellung eines Teils des Abschnitts 3 des 1. Kapitels bedeutete. Daraus muß man den Schluß ziehen, daß Marx sich erst während der Abfassung dieses Abschnitts 3 des 1. Kapitels über die Abschnittsgliederung: ,3) Die Productionszeit”, „Zweites Capitel. Der Umschlag des Capitals” klar wurde.

Überhaupt ist es fraglich, ob Marx schon vor Beginn der Abfassung des "Manuskripts I" den Plan des 2. Kapitels „Der Umschlag des Capitals" gehabt hatte. Auf Seite 28 schreibt er: „D. Wichtigkeit dieser Bestimmung zeigt sich beim Umschlag d. Capitals. ( $\$ 3$ dieses Capitels)"18; was vollkommen der Tatsache entspricht, daß er über Abschnitt 3 des 1. Kapitels zunächst „Der Umschlag des Capitals” schrieb. Es ist schwer vorstellbar, daß Marx einmal den Plan gehabt hätte, dieselbe Uberschrift ,Der Umschlag des Capitals" erst dem Abschnitt 3 des 1. Kapitels, dann noch einmal dem 2. Kapitel zu geben. Marx entschied sich demnach frühestens in dem Moment der Änderung der Überschrift des Abschnittes 3, das 2. Kapitel „Der Umschlag des Capitals” zu überschreiben. Kurz, es ist fast undenkbar, daß Marx vor Beginn des „Manuskripts I” den oben dargestellten detaillierten Plan im Kopf gehabt hätte.

Zweitens. Im „Manuskript I" ist die Überschrift des Abschnitts 1 des 1. Kapitels nur „Die Metamorphosen des Capitals”, im Plan dagegen „Die Metamorphosen des Kapitals: Geldkapital, Produktives Kapital, Waarenkapital". Man könnte meinen, daß Marx einfach Einzelheiten weggelassen hat. Wenn man aber sorgfältig den Abschnitt 1 des 1 . Kapitels des „Manuskripts I” liest, kann man leicht erkennen, daß Marx gerade während des Schreibens dieses Abschnitts die drei Formbestimmungen, besonders die des Warenkapitals, Schritt für Schritt herausarbeitete.

Im ,Manuskript I” gibt Marx als zweite der Kreislaufsformen die an, worin das Kapital von den Produktionselementen ausgeht und in die Produktionselemente zurückgeht.

1)

Zweiter Kreislauf: W. (D. Waare in d. Form d. Produktionsmittel.)

2) 3)

W. im Proceß. D. unmittelbare Productionsproceß. $-W^{\prime}-G \cdot W$. D. Ver- 
wandlung d. producirten Waaren in Geld $u$. ihre Rückverwandlung in Waare. (Die Faktoren d. Arbeitsprocesses.)
1) 2)
3)

Also W. - D. Proceß. - W'-G-W. ${ }^{19}$

Diese Form verschwand in den folgenden Manuskripten des II. Buches. $\mathrm{Daß}$ Marx im „Manuskript I” diese Form aufstellte, hängt damit zusammen, daß er W von G-W(Pm[ + A]), Waarencapital” nannte.

[...] d. Waarencapital erscheint doppelt als $\mathrm{W} u$. als $\mathrm{W}^{\prime}$, als Voraussetzung d. Processes $u$. als sein Resultat, als avance $u$. reprise. ${ }^{20}$

W, d. Waarencapital, worin sich d. Geldcapital verwandelt hat, drückt d. realen Faktoren d. Arbeitsprocesses, also d. stofflichen Existenzbedingungen v. W' aus, dem Waarencapital, wie es als Resultat aus d. Productionsproceß hervorgeht, $u$. wie es d. zweite Phase d. Circulation d. Capitals zu durchlaufen hat. ${ }^{21}$

Marx dürfte jedoch über diese Auffassung nachgedacht haben. Auf Seite 29 formulierte er schon deutlich den Unterschied der drei Formen des Kapitals.

Waarencapital u. Geldcapital sind d. beiden Formen d. Capitals, die es innerhalb d. eigentlichen Circulationssphäre unterscheiden v. sich als productives Capital, seiner Existenzform innerhalb d. eigentlichen Productionssphäre. Beide Formen sind hier etwas näher zu bestimmen. ${ }^{22}$

Danach erörterte er in erster Linie unter dem Titel „Waarencapital. W'-G” die charakteristische Bestimmung von $W^{\prime}$. Und jetzt schreibt er:

Waarencapital im eminenten Sinn ist also das ausschließlich in d. Form d. v. ihm producirten Waare u. als Waare functionirende Capital, d.h. d. Capital in einer Form, worin es als Waare verkauft werden, (seine Geldform hergestellt mit Bezug auf es als vorgeschossenes Capital), seine erste Metamorphose durchmachen muß. ${ }^{23}$

Anschließend zeigt er schon nur drei Kreislaufsformen auf, d.h. den Kreislauf des Geldkapitals, des produktiven Kapitals und des Warenkapitals, ohne jene Form, W-P-W'-G'-W, anzugeben. ${ }^{24}$ Nach der Darstellung dieses Abschnitts kann man vermuten, daß Marx sich gerade auf Grund dieser Darstellung entschied, der Überschrift „Die Metamorphosen

19 Manuskriptseite 4, ebd., 1. 3 verso. Danach fuhr Marx Seiten 18-19 diese Form aus, ebd., 1.10 verso, 11.

20 Manuskriptseite 5, ebd., 1. 4.

21 Manuskriptseite 6, ebd., 1. 4 verso.

22 Ebd., 1. 16.

23 Manuskriptseite 32, ebd., 1.17 verso.

24 Ebd. 
des Capitals” noch „Geldkapital, Produktives Kapital, Waarenkapital” hinzuzufügen.

Drittens. Es gibt noch deutlichere Anhaltspunkte. Das sind einmal die Überschriften im ,Manuskript IV" und in jenem davor geschriebenen Fragment. Im Fragment schrieb Marx zunächst die folgende Úberschrift:

Zweites Buch. Der Cirkulationsprozeß des Kapitals.

Erstes Kapitel. Die Cirkulation des Kapitals.

1) Die Metamorphosen des Kapitals. ${ }^{25}$

In der zweiten Hälfte dieser Seite begann er nochmals von vorn.

1) Die Metamorphose des Kapitals: Geldkapital, Waarenkapital, Productives Kapital. ${ }^{26}$

Anschließend schrieb Marx im Text des „Manuskripts IV” folgende Überschriften:

Zweites Buch. Der Cirkulationsprozeß des Kapitals.

Erstes Kapitel. Der Umlauf des Kapitals.

1) Die Metamorphosen des Kapitals: Geldkapital, Produktives Kapital, Waarenkapital.

2) Produktionszeit und Umlaufszeit.

3) Die Cirkulationskosten.

Zweites Kapitel. Der Umschlag des Kapitals.

1) Begriff des Umschlags.

2) Fixes Kapital und Cirkulirendes Kapital. (Anlagekapital u. Betriebskapital. $)^{27}$

Es ist leicht zu erkennen, daß diese Überschriften im Prinzip denen in dem Plan des II. Buches entsprechen, nur daß hier im Abschnitt 2 des 2. Kapitels in der Klammer statt „Umschlagscyclen” „Anlagekapital u. Betriebskapital" steht.

Aber es sind noch einige Bemerkungen notwendig. Die Überschrift des 1. Kapitels lautet im Plan „Die Cirkulation des Kapitals”, im „Manuskript IV” hingegen „Der Umlauf des Kapitals”. Die letztere Überschrift scheint auf eine Beibehaltung der Formulierung im „Manuskript l” hinzudeuten. In Wirklichkeit hatte Marx aber zunächst „Die Cirkulation des Kapitals” geschrieben; erst im nachherein hat er „Die Cirkulation” in „Der Umlauf” geändert. Also ist die Reihenfolge, in der Marx die Varianten der Úberschrift des 1. Kapitels in den betreffenden Manuskripten benutzt hat, folgende:

${ }^{25}$ Marx-Engels-Nachlaß, IISG, A 66 (im alten Inventar A 47), Manuskriptseite 1.

${ }^{26}$ Ebd.

27 Ebd., A 65. Manuskriptseiten 1, 30, 35, 50, 53. 
„Der Umlauf des Capitals”:

„Die Cirkulation des Kapitals”:

„Der Umlauf des Kapitals”:
„Manuskript I";

Plan;

Fragment zum ,Manuskript IV”;

erste Fassung im „Manuskript IV”;

zweite Fassung im ,Manuskript

IV".

Es ist also davon auszugehen, daß der Plan, das Fragment und das „Manuskript IV" unmittelbar nacheinander entstanden sind, wobei anzunehmen ist, daß der Plan vor dem ,Manuskript IV" geschrieben worden ist, a) deshalb, weil die Überschrift des Abschnitts 2 des 2. Kapitels des Plans der im ,Manuskript I" ähnlich ist; b) deshalb, weil, falls Marx den Plan nach dem „Manuskript IV” geschrieben hätte, er sich wohl bei diesem, aber nicht bei dem „Manuskript I”, wo er jetzt ist, ${ }^{28}$ befände.

Es drängt sich hier die Frage auf, warum Marx im Plan des II. Buches weder die Überschrift noch den Inhalt des 3. Kapitels angegeben hat; denn die vier Autoren schreiben: „Das dritte Kapitel erhielt bei der Herstellung des ,Manuskripts I' des II. Buches den Titel ,Zirkulation und Reproduktion'; gleichzeitig erarbeitete Marx die Struktur dieses Kapitels." ${ }^{29}$ Man kann hier mancherlei vermuten, sollte aber in Betracht ziehen, daß Marx schon auf der letzten Seite des ,Manuskripts I" den Plan für das 3. Kapitel niedergelegt hatte.

Das „Manuskript I” des II. Buches und das „Hauptmanuskript” des III. Buches

Das Argument der vier Autoren dafür, daß Marx die Arbeit am III. Buch frühestens auf Seite 256 unterbrach, ist: Auf Seite 256 verweist Marx auf den Abschnitt 3 im 1. Kapitel des ,Manuskripts I”, der den Zirkulationskosten gewidmet ist, was zwar der Abschnittsgliederung des Plans des II. Buches, nicht aber der im Text des „Manuskripts I” entspricht. Da der Plan vor dem „Manuskript I" geschrieben worden sei, beweise dieser Verweis, daß nur der Plan, aber noch nicht das „Manuskript I" des II. Buches vorlag, Marx das „Manuskript I" also frühestens nach Seite 256 anfing. ${ }^{30}$ Jetzt müssen wir dagegen sagen: Da das ,Manuskript I" vor dem Plan geschrieben worden ist, beweist dieser Verweis, daß Marx auf Seite 256 außer der Abschnittsgliederung im Text des „Manuskripts I” - „Erstes

28 Die vier Verfasser schreiben: „Der Plan für das II. Buch ist, auf einem Einzelblatt geschrieben. zusammen mit dem ,Manuskript I' des II. Buches erhalten geblieben." Miskewitsch, Ternowski, Tschepurenko, Wygodski, ,Zur Periodisierung”, S. 299.

29 Ebd., S. 300.

30 Ebd., S. 308 
Kapitel, 3) Umschlag des Capitals” bzw. ,Erstes Capitel, 3) Die Productionszeit" - schon die neue Abschnittsgliederung - „Erstes Kapitel, 3) Die Cirkulationskosten" - erarbeitet hatte, die im „Manuskript IV” (sogar noch im ,Manuskript II") erscheint.

Wenn der Plan des II. Buches nach dem „Manuskript I” geschrieben worden wäre, wären die Verweise auf den Seiten 243 und 256 des „Hauptmanuskripts” des III. Buches, die beweisen sollten, daß Marx hier das "Manuskript I" noch nicht geschrieben hatte, vielmehr ein Beweis dafür, daß Marx hier immerhin schon das 1. Kapitel des „Manuskripts I” geschrieben hatte. Wenn man von dem schwer vorstellbaren Fall absieht, daß Marx das „Manuskript I" des II. Buches und das „Hauptmanuskript” des III. Buches in einer gewissen Phase parallel geschrieben hat, muß man annehmen, daß vor der Seite 243 von diesem schon das ganze ,Manuskript I" des II. Buches geschrieben war.

Unbestreitbar scheint mir indessen die folgende Schlußfolgerung der vier Autoren:

Im II. Kapitel des III. Buches befindet sich auf Seite 164 folgende für unser Thema aufschlußreiche Bemerkung: ,Man erinnert sich aus dem Buch II, daß hier in der Zirkulationszeit die Produktionszeit eingeschlossen ist, indem jede Phase worin sich das Kapital befindet von der Verwandlung des Gelds in die stofflichen Elemente des Kapitals bis zur Rückverwandlung des Produkts in Geld (und daher auch bis zur Realisierung des Mehrwerts) eine Phase seiner Gesamtzirkulation oder seines Umschlags bildet. Wie weit die Zirkulationszeit auf die Profitrate einwirkt, Frage auf die wir hier nicht im Detail eingehen wollen [da Buch II noch nicht geschrieben, worin dies ex professo erörtert wird.]" Dies bestätigt ein übriges Mal, daß das ..Manuskript I" des II. Buches von Marx nach Abfassung des II. Kapitels des III. Buches geschaffen worden ist. ${ }^{31}$

Selbst wenn es den in eckigen Klammern gesetzten Satz nicht gäbe, könnte man zum gleichen Schluß gelangen; denn a) werden die „Zirkulationszeit” und die „Produktionszeit” schon im „Manuskript I” des II. Buches klarer behandelt und definiert, b) wird die Frage, wie weit die Zirkulationszeit auf die Profitrate einwirkt, namentlich in seinem 2. Kapitel ziemlich eingehend erörtert. Daraus können wir schließen, daß der Satz von Marx „Buch II noch nicht geschrieben” wirklich bedeutete, daß das „Manuskript I" noch nicht geschrieben war.

Einen ähnlichen Anhaltspunkt gibt es noch später. Auf Seite 182 des „Hauptmanuskripts” des III. Buches findet sich die folgende Bemerkung: "[D. Begriff d. Markts muß in s. allgemeinsten Zügen entwickelt werden in 
d. Abschnitt über d. Circulationsproceß des Capitals.]”32 Da der ,Begriff des Markts” auf Seite 32-33 des „Manuskripts I” des II. Buches gerade ,in seinen allgemeinsten Zügen entwickelt" ist, ${ }^{33}$ ist anzunehmen, daß hier das „Manuskript I" des II. Buches noch nicht vorlag.

Wir können also zusammenfassend sagen, daß Marx irgendwo nach Seite 182, aber vor Seite 243 die Arbeit am ,Hauptmanuskript” des III. Buches unterbrach und das „Manuskript I” des II. Buches schrieb.

${ }^{33}$ IML, f. 1, op. 1, d. 1802, 1. 17 verso, 18. 